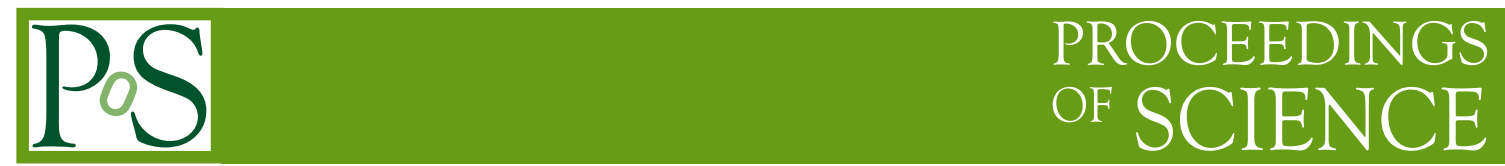

\title{
How to calibrate the jet energy scale?
}

\author{
Kenichi Hatakeyama*† \\ (Rockefeller University) USA \\ E-mail: hatakekerockefeller.edu
}

\begin{abstract}
Top quarks dominantly decay into $b$-quark jets and $W$ bosons, and the $W$ bosons often decay into jets, thus the precise determination of the jet energy scale is crucial in measurements of many top quark properties. I present the strategies used by the CDF and DØ collaborations to determine the jet energy scale. The various cross checks performed to verify the determined jet energy scale and evaluate its systematic uncertainty are also discussed.
\end{abstract}

International Workshop on Top Quark Physics

January 12-15, 2006

Coimbra, Portugal

\footnotetext{
* Speaker.

$\dagger$ on behalf of the CDF and DØ Collaborations
} 


\section{Introduction}

Many measurements of hard scattering processes at hadron colliders, including $t \bar{t}$ production, depend on the accurate determination of jet energies, since jets are used as measures of partons from the hard scattering. In particular, the top quark mass determination is limited by the uncertainty on the jet energy scale.

The determination of the jet energy scale is cumbersome because of instrumental effects, including calorimeter non-uniformity, resolution effects due to large fluctuations in particle showering in the calorimeter, the non-linear response of the calorimeter especially to hadrons, and different responses to different types of particles, e.g. hadrons versus electromagnetically interacting particles. Energy from additional $p \bar{p}$ interactions occurring in the same bunch crossing also comes into the jet cone, which has a non-negligible effect at low jet $p_{T}$ and high instantaneous luminosities. The situation is further complicated due to the fact that the underlying event contributes energy to the jet clustering cone, and that the jet cone does not contain all the energy of the parent parton because of parton shower. These effects have to taken into account, when the jet energy scale determination or validation based on the hadronic mass resonances and $p_{T}$ balance of jets with a photon or $Z$-boson is performed. The parton shower and jet fragmentation also depend on the flavor of the parent parton which results in the different jet energy correction.

$\mathrm{CDF}$ and $\mathrm{D} \emptyset$ use different approaches to determine the jet energy scale. At CDF, determination of the absolute jet energy scale relies primarily on the detector simulation and jet fragmentation model [1]. The calorimeter simulation has been tuned to reproduce the single particle response measured in the test beam data and $p \bar{p}$ collision data, and the jet fragmentation model in PYTHIA is used to simulate jets. The energy scale is validated by comparing the $\gamma$-jet and $Z$-jet $p_{T}$ balance observed in collision data with that in Monte Carlo (MC) events. In the approach used by DØ to determine the jet energy scale, the absolute jet response is obtained by utilizing the transverse momentum conservation in photon+jet events independently in data and MC events [2]. These are discussed in section 3 .

\section{Detector}

The CDF and $\mathrm{D} \emptyset$ detectors are general-purpose detectors for $p \bar{p}$ collisions at the Fermilab Tevatron collider. Both detectors comprise a solenoidal-magnetic charged particle spectrometer surrounded by the calorimeters and a set of muon chambers. The component most relevant for the measurement of jets is the calorimeter. The calorimeter is used to measure the energy of particles produced in $p \bar{p}$ collisions. The $\mathrm{CDF}$ and $\mathrm{D} \emptyset$ calorimeters both have a projected tower geometry.

The CDF calorimeter is a sampling calorimeter. The electromagnetic (EM) section consists of alternating layers of lead and scintillator, and the hadronic (HAD) section consists of alternating layers of steel and scintillator. The CDF calorimeters are divided into two main pseudorapidity $(|\eta|)$ regions; the central calorimeter covers $|\eta|<1.1$ and the plug region covers $1.1<|\eta|<3.6$. The region between the central and plug regions is covered by the end-wall hadron calorimeter. The tower size in the central region is $\Delta \eta \times \Delta \phi \approx 0.1 \times 15^{\circ}$, and the segmentation in the plug region varies as function of $\eta\left(\Delta \eta \times \Delta \phi \approx 0.1 \times 7.5^{\circ}\right.$ for $|\eta|<1.8$ and $\Delta \eta$ increases with increasing $\left.|\eta|\right)$. The noise level is very low, having $\sim 1$ noise tower with $E_{T}>50 \mathrm{MeV}$ per event. 
The $\mathrm{D} \emptyset$ calorimeters are uranium and liquid argon sampling calorimeters. The $\mathrm{D} \emptyset$ calorimeters consist of the central calorimeters covering $|\eta| \lesssim 1$ and the end calorimeters extend the coverage to $|\eta| \sim 4$. The calorimeters have three sections: EM, fine hadronic (FH) and coarse hadronic $(\mathrm{CH})$. In the $\mathrm{CH}$ section, copper or steel is used instead of uranium as a absorber. The DØ calorimeters are nearly compensating, with an $e / \pi$ ratio less than 1.05 above $30 \mathrm{GeV}$. The tower segmentation in $\eta-\phi$ space is $0.1 \times 5.625^{\circ}$.

\section{Energy scale determination for generic jets}

Jets (calorimeter jets) are reconstructed from energies measured in calorimeter towers using jet clustering algorithms. The jet algorithms used by the CDF and $D \emptyset$ collaborations are described elsewhere [3]. For measurements of the top quark properties, CDF and D $\varnothing$ use the cone-based jet algorithms. In MC simulation, particle jets can also be defined as jets reconstructed by applying the same jet clustering algorithm on stable final state particles. This section describes the procedures used by the $\mathrm{CDF}$ and $\mathrm{D} \emptyset$ collaborations to correct the calorimeter jet energy to that of the particle jet. The procedure used by CDF to correct jets back to the parent partons is also presented.

\subsection{Jet energy scale determination at $\mathrm{D} \emptyset$}

The jet energy calibration procedure employed by $\mathrm{D} \emptyset$ is based primarily on data, exploiting the transverse momentum conservation in events [2]. The measured jet energy is corrected back to the true particle jet energy by:

$$
E_{\text {jet }}^{\text {particle }}=\frac{E_{\text {jet }}^{\text {measured }}-E_{0}}{R_{\text {jet }} \cdot S},
$$

where $E_{0}$ is an offset energy which includes the underlying event, uranium noise, energy from the previous bunch crossing, and additional $p \bar{p}$ interactions. $R_{j e t}$ represents the calorimeter response to jets which corrects for the calorimeter non-uniformity along $\eta$ and then corrects the absolute energy scale. $S$ is the showering correction which corrects for energy emitted outside the jet cone due to detector effects.

\subsubsection{Offset}

The offset $E_{0}$ includes the underlying event, noise from the radioactive decay of the uranium absorber, the effect of the previous bunch crossing, and the contributions of additional $p \bar{p}$ interactions. The underlying event energy is defined as the energy contributed by spectator partons to the jets. The offset $E_{0}$ is determined by measuring the transverse energy density in the minimum-bias and zero-bias data.

\subsubsection{Response}

Even after the calorimeter energy calibration based on the test beam data, the jet energy measured by the calorimeter is less than the true jet energy on average due to non-linear response to low momentum particles, dead materials, and module-to-module fluctuations. In order to measure the calorimeter response to jets, DØ uses the missing $E_{T}$ projection fraction method which exploits the transverse momentum conservation in an event [2]. 
In photon+jet events, the transverse energies of the real photon and the other recoil particles at the particle level should satisfy:

$$
\vec{E}_{T}^{\gamma}+\vec{E}_{T}^{\text {recoil }}=0
$$

In general, the calorimeter response to both photons and recoils is less than unity and the energy mismeasurement causes the missing $E_{T}\left(\not_{T}\right)$ in events:

$$
R_{\gamma} \vec{E}_{T}^{\gamma}+R_{\text {recoil }} \vec{E}_{T}^{\text {recoil }}=-\vec{\not}_{T} .
$$

After the EM energy calibration, $R_{\gamma}=1$, and Eqs. (3.2), (3.3) transform to:

$$
R_{\text {recoil }}=1+\frac{\vec{E}_{T} \cdot \vec{n}_{T}^{\gamma}}{E_{T}^{\gamma}}
$$

where $\vec{n}_{T}^{\gamma}=\vec{E}_{T}^{\gamma} /\left|\vec{E}_{T}^{\gamma}\right|$. In back-to-back photon+jet events, $R_{\text {recoil }}$ can be considered as the response of a jet $R_{\text {jet }}$. In order to be less sensitive to jet energy resolution when determining the jet energy response, the study is performed as a function of $E^{\prime}=E_{T}^{\gamma} \cosh \left(\eta_{j e t}\right) . E_{T}^{\gamma}$ and $\eta_{\text {jet }}$ are better measured than $E_{j e t}$, and $E^{\prime}$ can be mapped to $E_{j e t}$. Figure 1 shows the jet response, $R_{j e t}$, as a function of $E_{j e t}$. In practice, the absolute jet response correction is determined and applied after the response is equalized with the following procedure.

The correction which equalizes the jet response in $\eta$ is derived from dijet events. In dijet events with one jet in the central region $(|\eta|<0.5)$ and the other jet anywhere in the detector, the relative jet response with respect to the central jet can be expressed as:

$$
R_{\text {jet }}=1+\frac{\vec{\not}_{T} \cdot \vec{n}^{\text {central jet }}}{E_{T}^{\text {central jet }}}
$$

and is measured as a function of the jet $\eta$. The correction is cross-checked for low $E_{T}$ jets in photon+jet events.

\subsubsection{Showering}

The showering correction accounts for the energy flow through the jet cone boundary during calorimeter showering. Due to electromagnetic and hadronic showering in the calorimeter, some particles produced inside the jet cone deposit part of their energy outside the cone. The showering correction corrects for this effect, but does not correct for the energy outside the jet cone due to gluon emission or fragmentation. The correction is derived by measuring the fraction of the energy outside the jet cone in the data and in the MC simulation at the particle level. The fraction measured in data includes both the loss due to the calorimeter showering and parton shower, while the fraction in $\mathrm{MC}$ at the parton level includes the loss due to parton shower only; thus the difference is used to determine the showering correction.

\subsubsection{Systematic uncertainty}

Figure 1 shows the systematic uncertainties associated with each step of the jet energy corrections and the total uncertainty for central jets as function of jet energy for D $\emptyset$. The jet response is the main source of the jet energy scale uncertainty over a wide range of jet energy; however, the offset and showering corrections give non-negligible contributions at low and high jet energies, respectively. 

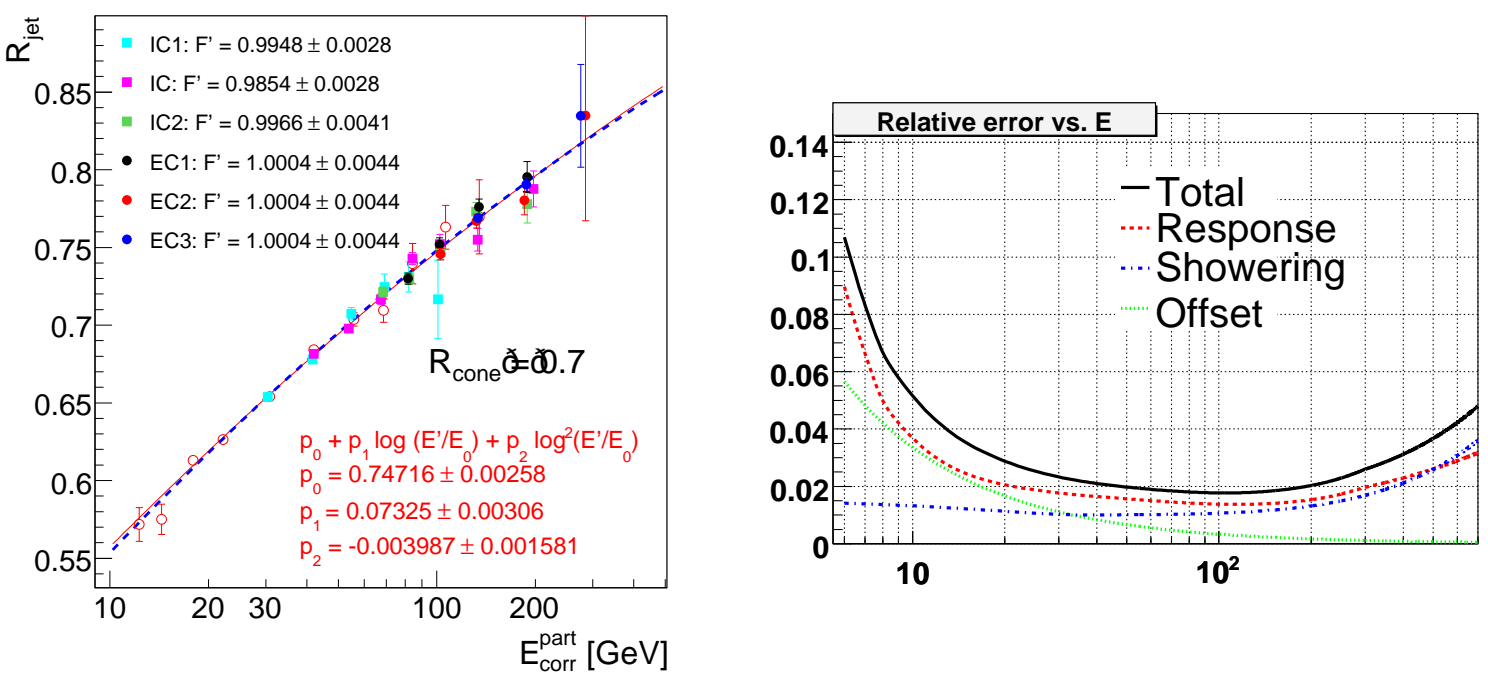

Figure 1: (left) $R_{\text {jet }}$ as a function of $E_{\text {jet }}$ measured in photon+jet events using the missing $E_{T}$ projection fraction method by DØ. (right) The systematic uncertainty for central jets as a function of jet energy at DØ.

\subsection{Jet energy scale determination at CDF}

At CDF, jets are corrected in steps as given in:

$$
p_{T, j e t}^{\text {particle }}=\left[p_{T, j e t}^{\text {measured }} \times f_{\text {rel }}-p_{T}^{M I} \times N_{v t x}\right] \times f_{a b s}, \quad p_{T}^{\text {parton }}=p_{T, j e t}^{\text {particle }}-p_{T}^{U E}+p_{T}^{O O C} .
$$

The jet $p_{T}$ measured by the calorimeters is scaled by $f_{\text {rel }}$ to make the jet response uniform with $\eta$. The $p_{T}$ from additional interactions in the same bunch crossing, $p_{T}^{M I}$, is subtracted based on the number of reconstructed primary vertices $N_{v t x}$ in an event. The jet $p_{T}$ is then corrected for the calorimeter response by the scale factor $f_{a b s}$, determined by matching particle jets with calorimeter jets. In some analyses, e.g. the top quark mass measurement, it is essential to determine the energy of the parent parton. Because of that, the underlying event and out-of-cone corrections, $p_{T}^{U E}$ and $P_{T}^{O O C}$, which correct jets back to parent partons are provided. This multi-step approach accommodates different needs in many different analyses at CDF.

\subsubsection{Calorimeter simulation}

At CDF, determination of the calorimeter jet energy scale primarily relies on a detector simulation and a jet fragmentation model. So, the accuracy of the calorimeter simulation is crucial for the precise jet energy scale determination. The CDF calorimeter response to single particles is tuned to reproduce the response measured in the test beam and the collision data. The CDF detector simulation uses a GEANT-based detector simulation in which a parametrized shower simulation (GFLASH) is used for the calorimeter response. Some of the GFLASH parameters are tuned to reproduce the single particle response ( $E / p$ and lateral profiles) measured in the test beam and collision data. Figure 2 shows the measured and simulated response for individual particles in the central region at CDF. The simulated response for electromagnetic particles (electrons, photons) agrees with the measured response to $1.7 \%$ at any momenta. The simulated response for charged hadrons agrees with the measured response within $2.5 \%$ for $p<12 \mathrm{GeV} / c$ and $3 \%$ for $12<p<20 \mathrm{GeV} / c$ 
$\widehat{\stackrel{Q}{\uplus}}$
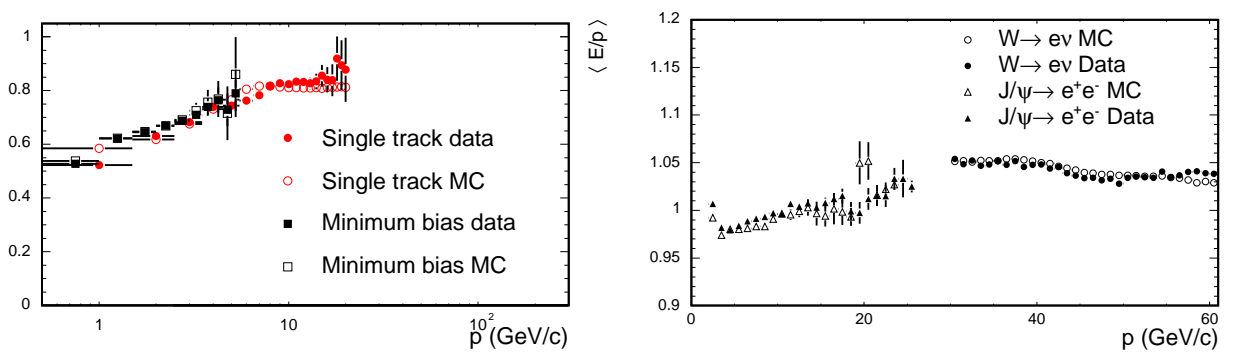

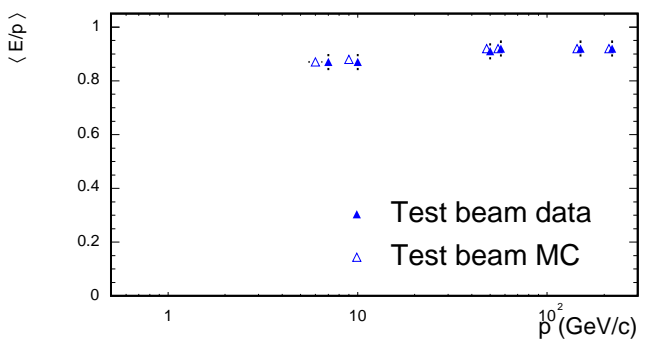

Figure 2: $\langle E / p\rangle$ versus $p$ for charged hadrons in the collision data (top left), for charged pions in the test beam data (bottom left), and for the electrons and positrons in the collision data (top right) compared to the simulation.

in the central region. At higher momenta, the uncertainty is $4 \%$ due to the test beam momentum uncertainty and the change in the integration time in the CDF data acquisition system.

The same method was used to tune the simulation response in the plug region. However, the tuning is limited in precision in the plug region because of the limited tracking coverage. Thus, the jet energy scale in the plug region is rescaled to that of the central region as discussed in the next section.

\subsubsection{Relative correction}

The CDF calorimeter response is not uniform in $\eta$ because of the boundaries between the calorimeter modules and the different responses of the central and plug calorimeters. The jet energy scale is made uniform in $\eta$ by scaling jet energies outside $0.2<|\eta|<0.6$ to those within this $\eta$ region where the energy scale is best understood. The correction is determined based on the $p_{T}$ balance of the leading two jets in dijet events where, to leading order, two jets should have the same $p_{T}$ and any imbalance is due to calorimeter non-uniformity. As discussed in the previous paragraph, tuning of the plug calorimeter is still limited in precision, so the relative correction is determined separately for the real and MC events. The two corrections are different by up to $\sim 2 \%$ at $|\eta|<2$.

\subsubsection{Multiple $p \bar{p}$ interaction correction}

Additional $p \bar{p}$ interactions in the same bunch crossing as the interaction which produced the jets contribute energy to the jet cone. The number of reconstructed primary vertices $\left(N_{v t x}\right)$ is a good estimator of the number of interactions in the same bunch crossing. This multiple interaction correction, $p_{T}^{M I}$, is derived by measuring the transverse momentum in a cone of the same size as the jet cone in the $0.2<|\eta|<0.6$ region as a function of $N_{v t x}$ in minimum bias events. $p_{T}^{M I}$ is 0.34 $\mathrm{GeV} / c$ for cone-size $R=0.4$ jets used in the top-quark analyses at CDF. 


\subsubsection{Absolute correction}

The absolute correction corrects calorimeter jets to the corresponding particle jets. The calorimeter simulation has been optimized to reproduce the measured single particle response, and it allows CDF to rely on the simulation to derive the absolute correction over a wide range of jet $p_{T}$.

The correction is obtained from dijet events generated with PүтніA 6.2 with tune A parameters [4]. Tune A refers to the values of the parameters describing multiple-parton interactions and initial state radiation which have been adjusted to reproduce the energy observed in the region transverse to the leading jet in jet data from CDF Run 1. It has been shown to provide a reasonable description of the measured energy distribution inside a jet [5]. The correction is determined by matching the two leading particle jets to the corresponding calorimeter jets and taking the most probable value of calorimeter-jet $p_{T}$ for particle jets with a given $p_{T, j e t}^{\text {particle }}$. This procedure takes out the jet energy smearing and falling spectrum effects from the correction.

The uncertainty of the absolute correction arises from the accuracy of the calorimeter simulation, calorimeter response time dependence, and the uncertainty in the jet fragmentation modeling. The resulting total uncertainty is $\sim 2 \%$ at $20 \mathrm{GeV} / c$ and $\sim 3 \%$ at $500 \mathrm{GeV} / c$, and is dominated by the calorimeter simulation uncertainty; further tuning is in progress.

\subsubsection{Underlying event and out-of-cone corrections}

It is often preferable to reconstruct the energy of the original parton rather than the energy of the particle jet. The conversion from the particle jet to the parent parton has to account for the out-of-cone (OOC) energy, which is the energy of the parent parton emitted outside the jet cone due to final state gluon radiation, and the underlying event (UE) energy from spectator partons. The correction was determined using the same method as the absolute correction, but the correction relates a particle jet with the matched parent parton.

The uncertainty of this correction arises from the modeling of UE and OOC energies. The OOC energy uncertainty is evaluated by comparing the energy flow outside the jet cone up to $R=1.3$ in data and MC. We measured in PYTHIA MC that an additional energy $0.5 \mathrm{GeV}$ falls outside a cone of 1.3 , and half of it is taken as the systematic uncertainty.

The uncertainty of the UE energy modeling is evaluated based on the UE study at CDF [6] that measured the energy transverse to the leading jet in dijet events in data and $\mathrm{MC}$ samples.

\subsubsection{Systematic uncertainties and cross-checks}

CDF has determined the jet energy scale using the procedure described above. In the central region, the current uncertainty on the jet scale is $6.5 \%$ at $20 \mathrm{GeV} / c$ dominated by the OOC energy uncertainty, $3 \%$ at $100 \mathrm{GeV} / c$ and $3.5 \%$ at $500 \mathrm{GeV} / c$ where it is dominated by the calorimeter simulation uncertainty.

The photon-jet and $Z$-jet data are good samples for studying the jet energy scale since the photon and $Z$ energies are well measured and thus provide good references for the jet energy. At $\mathrm{CDF}$, the photon-jet and $Z$-jet $p_{T}$ balances are used to cross-check the jet energy scale in data and MC events. The photon-jet $p_{T}$ balances in data, Pythia and Herwig MC events after all the corrections described above are shown in figure 4 . Only clean back-to-back photon+jet events with $\Delta \phi(\gamma, j e t)>3(\mathrm{rad})$ and the second jet $p_{T}<3 \mathrm{GeV} / \mathrm{c}$ are used. PythIA and HeRWIG MC events 


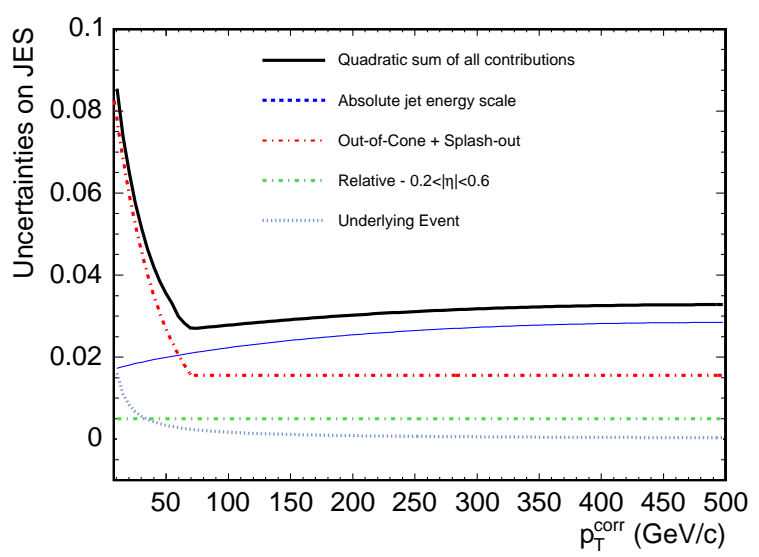

Figure 3: Jet energy scale uncertainties evaluated by CDF as a function of the corrected jet $p_{T}$ in $0.2<$ $|\eta|<0.6$.
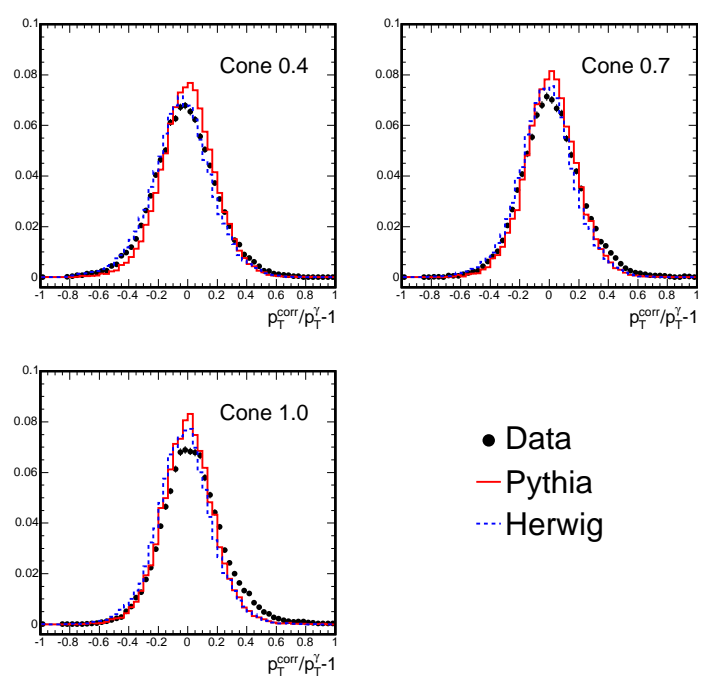

- Data

- Pythia

-...Herwig

Figure 4: Photon-jet $p_{T}$ balance in data (closed black circles), PYTHIA (solid red line) and HERWIG (dashed blue line) for $R=0.4$ (top left), 0.7 (top right) and 1.0 (bottom left) after all the corrections.

disagree by $\sim 3.5 \%$ for $R=0.4$ jets; however, data lie between PYTHIA and HERWIG and agree with both of them to within $2 \%$.

The hadronic decays of resonances with well known masses such as the $W$ and $Z$ bosons can be good places to test and calibrate the jet energy scale. Usually, the hadronic $W$ and $Z$ decays are swamped by QCD background at hadron colliders; however, in $t \bar{t}$ events with the lepton+jets topology, the hadronic $W$ resonance can be observed with a relatively small QCD background. Figure 5 shows the dijet mass distributions in four subsamples of the letpton+jet event sample; the subsamples are made based on the number of jets identified as $b$-quark jets by the standard CDF $b$-tagging algorithm [7]. Events with one $b$-tagged jet are further divided into two classes; 1 -tag(T) refers to events with four jets with $E_{T}>15 \mathrm{GeV}$ and 1-tag(L) refers to events with three jets with $E_{T}>15 \mathrm{GeV}$ and the fourth jet with $8<E_{T}<15 \mathrm{GeV}$.

The hadronic $W$-boson decay has been used successfully by the CDF and $\mathrm{D} \emptyset$ collaborations to 


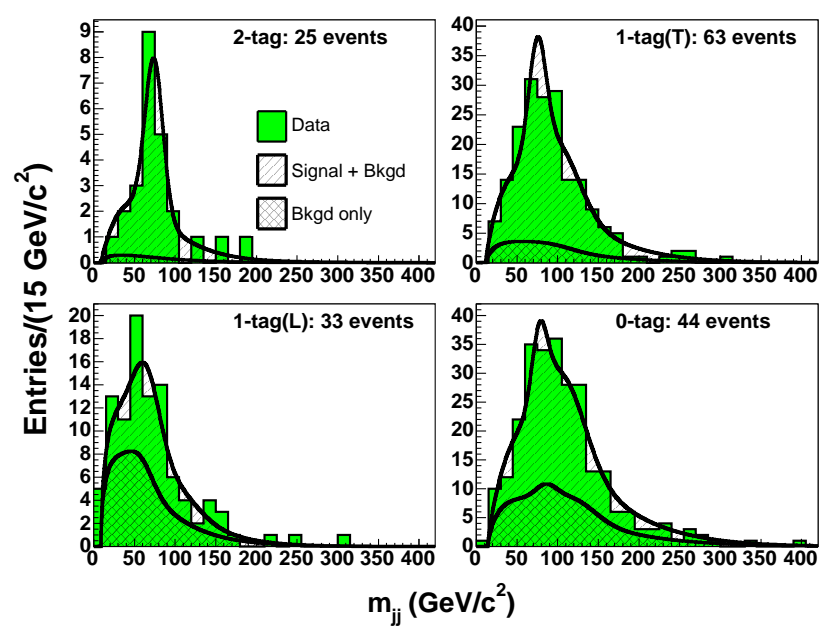

Figure 5: Dijet mass distributions for four subsamples in the lepton+jets sample. The signal and background shapes corresponding to the best fit of the jet energy scale cross-check are overlaid on the histograms.

calibrate the jet energy scale in top quark mass measurements in the lepton+jets channel [8, 9]. In the CDF measurement [8], the reconstructed top mass and dijet mass distributions are formed from $t \bar{t}$ MC events with various top mass and the jet energy scale ranging from -3 to $+3 \sigma$ where $\sigma$ is the total jet energy scale uncertainty described above. Fits to the data without using the jet energy scale constraint from the standard procedure yield the jet energy scale $[-0.25 \pm 1.22] \sigma$, indicating that the jet energy scale from the aforementioned procedure is in good agreement with information provided by the $W$ resonance peak in $t \bar{t}$ events.

Constraining the jet energy scale with the hadronic $W$ resonance is a very powerful technique, and the jet energy scale uncertainty from this method will improve as more data is accumulated. However, it has to be noted that all the detailed studies presented above would be crucial for the success of this technique, since this method relies on good modeling of the dijet mass distribution. Also, this technique would not be able to constrain the jet energy scale over a wide range of jet $p_{T}$.

\section{Energy scale for b-quark jets}

Top quarks decay dominantly to $b$-quarks and $W$ bosons, therefore it is essential to measure the energy response of $b$-quark jets ( $b$-jets) precisely. The correction from calorimeter jets to particle jets or to the parent parton is different for $b$-jets than for generic jets, since $b$-jets have different parton shower and fragmentation properties. Currently, both CDF and DØ rely on MC simulation to model $b$-jet energy scale.

In the CDF top quark mass measurements [8], additional uncertainties are evaluated for the $b$-jet energy scale: 1) uncertainties in energy response arising from uncertainty in $B$ meson semileptonic branching ratios, 2) uncertainties arising from the imperfect knowledge of the fragmentation properties of $b$-quarks, and 3) uncertainties arising from the different color flow associated with $b$-jets produced in top quark decay. The $b$-jet energy scale uncertainties from these sources are evaluated by changing the relevant parameters in MC based on the constraints from other experiments, and yield additional $0.6 \%$ uncertainty in total. 
Possible ways to test the $b$-jet energy scale in $p \bar{p}$ data would be to look at photon- $b$-jet $p_{T}$ balance or $Z \rightarrow b \bar{b}$ resonance. CDF has extracted the $Z \rightarrow b \bar{b}$ signal and is seeking to use it to test and calibrate the $b$-jet energy scale. At CDF, a dedicated trigger was implemented to collect a large sample of $Z$ decays to $b$-quark pairs by requiring two tracks with displaced vertices and two jets, and dijet events with both jets being tagged as $b$-jets are selected offline. The signal was extracted by fitting the QCD background shape computed using untagged data passing the same kinematic selection together with the $Z \rightarrow b \bar{b}$ signal and the $Z \rightarrow b \bar{b}$ signal shape computed with PYTHIA MC to the data. Currently, the systematic uncertainties associated with the QCD background subtraction procedure are being evaluated and procedures to test and calibrate the relative data/MC $b$-jet energy scale are being examined.

\section{Summary}

The strategies used by the CDF and DØ collaborations to determine the jet energy scale are presented. CDF has determined the jet energy scale primarily based on a tuned calorimeter simulation and jet fragmentation model, and the determined energy scale is validated in several cross checks. CDF has achieved a determination of the jet energy scale with the uncertainty shown in figure 3 [1] and is currently working to reduce it further. In the approach used by $D \emptyset$, the jet energy scale has been obtained mainly by utilizing the transverse momentum conservation in photon+jet events [2] and several cross-checks have been performed. DØ has determined the jet energy scale to within $\sim 2 \%$ precision over a wide range of jet $p_{T}$ (figure 11).

Hadronic $W$ decays in $t \bar{t}$ events with the lepton+jets topology have been successfully used to constrain the jet energy scale in the top quark mass measurements [8, 9$]$. This is a very powerful technique, however having excellent calorimeter simulation and well-calibrated jet energy response would still be crucial to make this work.

$b$-quark jets have different properties than generic jets, and the energy correction has to be determined separately. Both CDF and D $\varnothing$ are currently modeling the $b$-jet energy response based on $\mathrm{MC}$, and are currently investigating possibilities to test and calibrate the $b$-jet energy scale using $Z \rightarrow b \bar{b}$ and photon- $b$-jet $p_{T}$ balance.

\section{References}

[1] A. Bhatti et al., hep-ex/0510047, to be sumitted to Nucl. Instrum. Meth. A.

[2] B. Abbott et al. (D0 Collaboration), Nucl. Instrum. Meth. A 424, 352 (1999).

[3] G.C. Blazey et al., hep-ex/0005012.

[4] R. Field, "Herwig, Jimmy and PYTHIA Tune A", talk given at TeV4LHC at Fermilab (2004).

[5] D. Acosta et al. (CDF Collaboration), Phys. Ref. D 71, 112002 (2005).

[6] D. Acosta et al. (CDF Collaboration), Phys. Rev. D 70, 072002 (2004).

[7] C. Neu (for the CDF and DØ Collaborations), this proceeding.

[8] A. Abulencia et al. (CDF Collaboration), Phys. Rev. Lett. 96, 022004 (2006).

[9] V.M. Abazov et al. (DØ Collaboration), DØ-note 4874-CONF. 